\title{
Research on a Risk Assessment Method considering Risk Association
}

\author{
Zhan Zhang, ${ }^{1}$ Kai Li, ${ }^{1}$ and Lei Zhang ${ }^{2}$ \\ ${ }^{1}$ School of Business Administration, Northeastern University, Shenyang, China \\ ${ }^{2}$ School of Economics and Management, Beijing Jiaotong University, Beijing, China
}

Correspondence should be addressed to Lei Zhang; zhanglei_neu@sina.com

Received 26 August 2016; Revised 12 October 2016; Accepted 10 November 2016

Academic Editor: Yan-Wu Wang

Copyright @ 2016 Zhan Zhang et al. This is an open access article distributed under the Creative Commons Attribution License, which permits unrestricted use, distribution, and reproduction in any medium, provided the original work is properly cited.

Regarding risk assessment problems with multiple associated risks, a risk assessment method (RAM) is proposed in this paper. According to the risk-associated assessment information offered by expert panel, a comprehensive associated matrix is constructed to identify the influence relationship among risks so as to determine the hierarchical structure of risks. Then, based on the determined divided or undivided risk hierarchical structure as well as the possibility and loss of risks provided by expert panel, each value at risk (VAR) is calculated through knowledge related to probability theory. Finally, the feasibility and efficiency of the proposed method are demonstrated through a calculating case.

\section{Introduction}

Risk assessment is quantifying the probable degree of influence or loss brought by a certain event or thing [1]. Risk assessment is an important segment of risk management, for instance, in financial risk management, credit risk management, engineering risk management, and other aspects; it plays a significant decision-support role for risk managers to adopt reasonable risk prevention measures and strategies [24]. Over the years, many scholars at home and abroad have attached great importance to researches on RAM and there have already been some outstanding research achievements [5-10], like analytic hierarchy process about risk assessment $[5,6]$, hazard degree calculation method $[7,8]$, risk matrix method [9-11] and artificial neural network [12, 13], and so on. Though all the RAMs mentioned above have solved various kinds of risk assessment problems from different perspectives, most of them do not take risk-associated situations into account. However, in reality there are usually connections among risks. For example, Baranoff and Sager [14] analyzed the associated situation between resource risk and production risk in life-insurance companies. Abdellaoui et al. [15] find that associated risks are valued differently than corresponding reduced simple risks. Thus, research on
RAM in consideration of risk association has academic and practical application values. Now, there are few researches of this aspect. Liao et al. [16] adopted Bayesian Network approach to evaluate IT outsourcing risk meanwhile considering the association between IT outsourcing risk factors and IT outsourcing risks; Büyüközkan and Ruan [17] proposed a Choquet integrals-based software development risk evaluation approach regarding the association among development environment risk, code constraint risk, and engineering risk during the development of software. However, these RAMs mainly focus on specific risk assessment problems in risk-associated situations with no universal RAM proposed. Therefore, based on previous researches, we establish outsourcing risk hierarchy, introduce the interaction between different levels of risk, and give a RAM in consideration of multiple risk-associated situations.

There are four sections in this paper. In Section 1 we elaborate the research background and the problems that need to be explored or studied and then clarify the objectives and significance of the proposed method. In Section 2 we describe the RAM considering risk association in detail. In Section 3 we use a calculating case to prove feasibility and efficiency of the proposed method. Finally in Section 4 we summarize the conclusion and the main contributions of this 
paper and also the limitations and further research work to be carried out.

\section{Theories and Methods}

2.1. Description of Problems. In risk assessment problems, the occurrence of a certain risk may lead to another risk, that is, the association among risks. The following symbols are used to express the set and quantity of a risk assessment problem in consideration of risk-associated situations:

(i) $R=\left\{R_{1}, R_{2}, \ldots, R_{m}\right\}$ : the set of risks, where $R_{i}$ means the risk number $i, i=1,2, \ldots, m$

(ii) $E=\left\{E_{1}, E_{2}, \ldots, E_{n}\right\}$ : the set of the expert panel, where $E_{k}$ means the expert number $k, k=1,2, \ldots, n$

(iii) $w=\left(w_{1}, w_{2}, \ldots, w_{n}\right)$ : the weight vector of experts, where $w_{k}$ means the importance or weight of the expert $E_{k}$; it satisfies $0 \leq w_{k} \leq 1, \sum_{k=1}^{n} w_{k}=1, k=$ $1,2, \ldots, n$

(iv) $A_{k}=\left[a_{i j}^{k}\right]_{m \times m}$ : risk-associated matrix offered by the expert $E_{k}$, where $a_{i j}^{k}$ means the evaluation value offered by the expert $E_{k}$ for the direct influence degree of the risk $R_{i}$ on the risk $R_{j}$, in which five-point scale is adopted. 0 means "no influence," 1 means "weak influence," 2 means "rather weak influence," 3 means "medium influence," 4 means "rather strong influence," and 5 means "strong influence"

(v) $\lambda_{k}$ : the thresholds offered by the expert $E_{k}$ for comprehensive influence degree. Setting up thresholds is to reject the influence relationship that is insignificant and has little comprehensive influence degree according to expert panel. $0 \leq \lambda_{k} \leq 1, k=1,2, \ldots, n$

(vi) $P_{i}^{k}$ : the probability of occurrence of the risk $R_{i}$ offered by the expert $E_{k}, k=1,2, \ldots, n, i=1,2, \ldots, m$

(vii) $l_{i}^{k}$ : the loss after the occurrence of the risk $R_{i}$ offered by the expert $E_{k}, k=1,2, \ldots, n, i=1,2, \ldots, m$

(viii) $P_{i}^{G}$ : the comprehensive probability of the occurrence of the risk $R_{i} . i=1,2, \ldots, m$

(ix) $L_{i}^{G}$ : the comprehensive loss of the risk $R_{i}, i=$ $1,2, \ldots, m$

(x) $z_{i}$ : the VAR of the risk $R_{i}, i=1,2, \ldots, m$

We propose the detailed procedure of RAM in consideration of risk association. Firstly, several risk-associated matrixes offered by expert panel are combined as group riskassociated matrix, while several thresholds of comprehensive influence degree offered by expert panel are combined as group threshold; secondly, risk relationship identification is performed through methods like matrix transform and there are usually two situations, divided and undivided risk hierarchical structures in the result of risk relationship identification. If the undivided risk hierarchical structure is set as situation $\mathrm{A}$, each value at risk would be calculated according to the occurrence probability and loss of each risk offered by the expert panel; if the divided risk hierarchical structure is set as situation $\mathrm{B}$, the expert panel are required to offer the occurrence probability of bottom risk and conditional probability according to the risk hierarchical structure. Furthermore, the comprehensive probability of the occurrence of each risk would be calculated through conditional probability formula and total probability formula specifically so as to calculate each value at risk in consideration of loss of each risk.

2.2. Risk Relationship Identification. According to the description of problems above, identification methods of relationship among several risks (or risk hierarchical structures) are listed below.

Firstly, $n$ risk-associated matrixes $\left(A_{1}, A_{2}, \ldots, A_{n}\right)$ are combined as a group-associated matrix $A_{G}=\left[a_{i j}^{G}\right]_{m \times m}$, among which the calculating formula of $a_{i j}^{G}$ is

$$
a_{i j}^{G}=\left\{\begin{array}{ll}
\sum_{k=1}^{n} a_{i j}^{k} w_{k}, & t_{i j} \leq \frac{n}{2} \\
0, & t_{i j}>\frac{n}{2}
\end{array} \quad i, j=1,2, \ldots, m .\right.
$$

In formula (1), $t_{i j}$ means the number of experts who score the direct influence degree of the risk $R_{i}$ on the risk $R_{j}$ as 0 . Furthermore, the group-associated matrix $A_{G}$ is transformed into regulated group-associated matrix $B_{G}=\left[b_{i j}^{G}\right]_{m \times m}$, among which the calculating formula of $b_{i j}^{G}$ is

$$
b_{i j}^{G}=\frac{a_{i j}^{G}}{\max _{i}\left\{\sum_{h=1}^{n} a_{i h}^{G}\right\}} \quad i, j=1,2, \ldots, m .
$$

Secondly, comprehensive influence matrix with indirect association $C=\left[c_{i j}\right]_{m \times m}$ is set up and the calculating formula is

$$
C=B_{G}\left(I-B_{G}\right)^{-1} \text {. }
$$

Meanwhile, $n$ thresholds $\left(\lambda_{1}, \lambda_{2}, \ldots, \lambda_{n}\right)$ of comprehensive influence degree are combined as a group threshold $\lambda_{G}$, and the calculating formula is

$$
\lambda_{G}=\sum_{k=1}^{n} w_{k} \lambda_{k}
$$

According to the group threshold $\lambda_{G}$, the comprehensive influence matrix $C=\left[c_{i j}\right]_{m \times m}$ is transformed into $\lambda_{G}$-cut matrix $C_{\lambda}=\left[\alpha_{i j}\right]_{m \times m}$, where

$$
\alpha_{i j}=\left\{\begin{array}{ll}
1, & c_{i j} \geq \lambda_{G} \\
0, & c_{i j}<\lambda_{G}
\end{array} \quad i, j=1,2, \ldots, m .\right.
$$

Assume $D=\left[d_{i j}\right]_{m \times m}$ as $0-1$ comprehensive associated matrix, in which

$$
d_{i j}=\left\{\begin{array}{ll}
\alpha_{i j}+1, & i=j \\
\alpha_{i j}, & i \neq j
\end{array} \quad i, j=1,2, \ldots, m .\right.
$$


Matrix $D$ reflects the preference of the expert panel for influence degree among each risk with indirect influence relationship. The influence of the risk $R_{i}$ on itself is 1 .

According to matrix $D$, hierarchical structure of each risk is divided. Assume

$$
H_{i}=H_{i} \cap J_{i} \quad i=1,2, \ldots, m,
$$

where $H_{i}=\left\{R_{j} \mid d_{j i}=1\right\}$ and $J_{i}=\left\{R_{j} \mid d_{i j}=1\right\} . H_{i}$ means the risk set corresponding to the element valued 1 in the list number $i$ in the matrix $D ; J_{i}$ means the risk set corresponding to the element valued 1 in the row number $i$ in the matrix $D$.

If formula (7) works for a certain $i, R_{i}$ should be regarded as the bottom element in $D$ and the list number $i$ and the row number $i$ in $D$ should be deleted to form a new matrix. During the process, if the bottom element cannot be found, risk assessment should be performed regarding situation $\mathrm{A}$. If there is bottom element, searching bottom element in the new matrix should be performed until all the elements in the matrix are deleted. Then the hierarchical structure of matrix $D$ should be built up according to the order of deleting. Finally, risk assessment should be performed for situation B.

2.3. Risk Assessment. RAMs for situations A and B are listed below.

Situation A. As for the situation that risk hierarchical structure cannot be divided, each risk is regarded as elements of the same layer to process. Firstly, the probability of occurrence of risk offered by the expert panel $\left(P_{i}^{1}, P_{i}^{2}, \ldots, P_{i}^{n}\right)$ is combined as comprehensive probability of occurrence of risk $P_{i}^{G}$, and the calculating formula is

$$
P_{i}^{G}=\sum_{k=1}^{n} w_{k} P_{i}^{k} \quad i=1,2, \ldots, m .
$$

Secondly, the loss of risks $\left(l_{i}^{1}, l_{i}^{2}, \ldots, l_{i}^{n}\right)$ offered by the expert panel is combined as comprehensive loss of risks $L_{i}^{G}$, and the calculating formula is

$$
L_{i}^{G}=\sum_{k=1}^{n} w_{k} l_{i}^{k} \quad i=1,2, \ldots, m .
$$

Finally, on the basis of the comprehensive probability of occurrence of risk $P_{i}^{G}$ and comprehensive loss of risks $L_{i}^{G}$, the value at risk is calculated, and the calculating formula is

$$
z_{i}=P_{i}^{G} L_{i}^{G} \quad i=1,2, \ldots, m
$$

Situation $B$. As for the situation that risk hierarchical structure can be divided, $R_{f}=\left\{R_{i 1}, R_{i 2}, \ldots, R_{i m}\right\}$ is assumed as the set of risk on the layer number $f$, and $R_{i f}$ is set as the risk number $i$ on the layer number $f, f=1,2, \ldots, m ; P_{k 1}=$ $\left(p_{1}^{k 1}, p_{2}^{k 1}, \ldots, p_{d}^{k 1}\right)$ is the probability vector of the occurrence of the risk on the first layer (the bottom layer) offered by the expert $E_{k}$, where $p_{j}^{k 1}$ is the probability of occurrence of the risk $R_{j}$ offered by the expert $E_{k} ; d_{f}$ is the number of risks on the layer number $f . R_{j, f-1}$ is assumed as the risk number $j$ on the layer number $f-1$ related to the risk $R_{i f}, \quad j=1,2, \ldots, d_{f-1}^{i f} ; d_{f-1}^{i f}$ is the number of risks on the layer number $f-1$ related to the risk $R_{i f}$. $R_{i f}=T$ is assumed as the occurrence of the risk $R_{i f}$, and $R_{i f}=F$ means that the risk $R_{\text {if }}$ does not happen. In consideration of two conditions, $R_{j, f-1}=T$ and $R_{j, f-1}=F$, there will be $2^{d_{f-1}^{i f}}$ conditional probabilities related to $R_{i f}=T$.

Furthermore, assuming that $P^{k}\left(R_{i f}=T \mid R_{1, f-1}=\right.$ $\left.T, R_{2, f-1}=T, \ldots, R_{d_{f-1}^{i f}, f-1}=T\right)$ is the probability of occurrence of the risk $R_{i f}$ offered by the expert $E_{k}$ with the risks $R_{1, f-1}, R_{2, f-1}, \ldots, R_{d_{f-1}^{i f}, f-1}$ happening simultaneously, $k=$ $1,2, \ldots, n ; P^{k}\left(R_{i f}=T \mid R_{1, f-1}=T, R_{2, f-1}=F, \ldots\right.$, $R_{d_{f-1}^{i f}, f-1}=T$ ) is the probability of occurrence of the risk $R_{i f}$ offered by the expert $E_{k}$ with the risks $R_{1, f-1}, R_{3, f-1}$, $R_{4, f-1}, \ldots, R_{d_{f-1}^{i f}, f-1}$ happening simultaneously but no risk $R_{2, f-1}, k=1,2, \ldots, n ; \ldots, P^{k}\left(R_{i f}=T \mid R_{1, f-1}=F, R_{2, f-1}=\right.$ $\left.F, \ldots, R_{d_{f-1}^{i f}, f-1}=F\right)$ is the probability of occurrence of the risk $R_{i f}$ without $R_{1, f-1}, R_{2, f-1}, \ldots, R_{d_{f-1}^{i f}, f-1}$ offered by the expert $E_{k}, k=1,2, \ldots, n$; for convenience, $P^{k}\left(R_{i f}=T\right.$ । $\left.R_{1, f-1}=T, R_{2, f-1}=T, \ldots, R_{d_{f-1}^{i f}, f-1}=T\right), P^{k}\left(R_{i f}=T\right.$ । $\left.R_{1, f-1}=T, R_{2, f-1}=F, \ldots, R_{d_{f-1}^{i f}, f-1}=T\right), \ldots, P^{k}\left(R_{i f}=\right.$ $\left.T \mid R_{1, f-1}=F, R_{2, f-1}=F, \ldots, R_{d_{f-1}^{i f}, f-1}=F\right)$ are recorded as $P^{k}\left(R_{i f}=T \mid T, T, \ldots, T\right), P^{k}\left(R_{i f}=T \mid\right.$ $T, F, \ldots, T), \ldots, P^{k}\left(R_{i f}=T \mid F, F, \ldots, F\right)$ for abbreviation.

Firstly, the probability vectors $P_{11}=\left(p_{1}^{11}, p_{2}^{11}, \ldots, p_{d}^{11}\right)$, $P_{21}=\left(p_{1}^{21}, p_{2}^{21}, \ldots, p_{d}^{21}\right), \ldots, P_{n 1}=\left(p_{1}^{n 1}, p_{2}^{n 1}, \ldots, p_{d}^{n 1}\right)$ are combined as the comprehensive probability vector $P_{1}=$ $\left(p_{1}^{1}, p_{2}^{1}, \ldots, p_{d}^{1}\right)$ and the calculating formula is

$$
p_{j}^{1}=\sum_{k=1}^{n} w_{k} p_{j}^{k 1} \quad j=1,2, \ldots, d_{1} .
$$

Secondly, the conditional probabilities $P^{1}\left(R_{i f}=T\right.$ । $T, T, \ldots, T), P^{2}\left(R_{i f}=T \mid T, T, \ldots, T\right), \ldots, P^{n}\left(R_{i f}=T \mid\right.$ $T, T, \ldots, T)$ are combined as the comprehensive conditional probability $P_{G}\left(R_{i f}=T \mid T, T, \ldots, T\right)$; the conditional probabilities $P^{1}\left(R_{i f}=T \mid T, F, \ldots, T\right), P^{2}\left(R_{i f}=T \mid T, F\right.$, $\ldots, T), \ldots, P^{n}\left(R_{i f}=T \mid T, F, \ldots, T\right)$ are combined as the comprehensive conditional probability $P_{G}\left(R_{i f}=T\right.$ । $T, F, \ldots, T) ; \ldots$; the conditional probabilities $P^{1}\left(R_{i f}=T\right.$ । $F, F, \ldots, F), P^{2}\left(R_{i f}=T \mid F, F, \ldots, F\right), \ldots, P^{n}\left(R_{i f}=T \mid\right.$ $F, F, \ldots, F)$ are combined as the comprehensive conditional probability $P^{n}\left(R_{i f}=T \mid F, F, \ldots, F\right)$, and the calculating formulas are as follows:

$$
\begin{aligned}
P_{G} & \left(R_{i f}=T \mid T, T, \ldots, T\right) \\
& =\sum_{k=1}^{n} w_{k} P^{k}\left(R_{i f}=T \mid T, T, \ldots, T\right),
\end{aligned}
$$




$$
\begin{gathered}
P_{G}\left(R_{i f}=T \mid T, F, \ldots, T\right) \\
=\sum_{k=1}^{n} w_{k} P^{k}\left(R_{i f}=T \mid T, F, \ldots, T\right), \\
\vdots \\
P_{G}\left(R_{i f}=T \mid F, F, \ldots, F\right) \\
=\sum_{k=1}^{n} w_{k} P^{k}\left(R_{i f}=T \mid F, F, \ldots, F\right) .
\end{gathered}
$$

According to formulas (11)-(12b $\left.\mathrm{d}_{f-1}^{i f}\right)$, total probability formula is adopted to calculate the comprehensive probability $P_{i}^{G}$ of occurrence of each risk specifically (from the bottom layer); for instance, the formula to calculate the comprehensive probability of occurrence of risk on the layer number $f$ is

$$
\begin{aligned}
P_{i}^{G} & =P_{G}\left(R_{1, f-1}=T, R_{2, f-1}=T, \ldots, R_{d_{f-1}^{i f}, f-1}\right. \\
& \left.=T, R_{i f}=T\right)+P_{G}\left(R_{1, f-1}=T, R_{2, f-1}\right. \\
& \left.=F, \ldots, R_{d_{f-1}^{i f}, f-1}=T, R_{i f}=T\right)+\cdots \\
& +P_{G}\left(R_{1, f-1}=F, R_{2, f-1}=F, \ldots, R_{d_{f-1}^{i f}, f-1}\right. \\
& \left.=F, R_{i f}=T\right),
\end{aligned}
$$

where

$$
\begin{aligned}
& P_{G}\left(R_{1, f-1}=T, R_{2, f-1}=T, \ldots, R_{d_{f-1}^{i f}, f-1}\right. \\
& \left.\quad=T, R_{i f}=T\right)=P_{G}\left(R_{1, f-1}=T\right) P_{G}\left(R_{2, f-1}\right. \\
& \quad=T) \cdots P_{G}\left(R_{i f}=T \mid R_{1, f-1}=T, R_{2, f-1}\right. \\
& \left.\quad=T, \ldots, R_{d_{f-1}^{i f}, f-1}=T\right), \\
& P_{G}\left(R_{1, f-1}=T, R_{2, f-1}=F, \ldots, R_{d_{f-1}^{i f}, f-1}\right. \\
& \left.\quad=T, R_{i f}=T\right)=P_{G}\left(R_{1, f-1}=T\right) P_{G}\left(R_{2, f-1}\right. \\
& \quad=F) \cdots P_{G}\left(R_{d_{f-1}^{i f}, f-1}=T\right) P_{G}\left(R_{i f}\right. \\
& \quad=T \mid R_{1, f-1}=T, R_{2, f-1}=F, \ldots, R_{d_{f-1}^{i f}, f-1} \\
& =T),
\end{aligned}
$$

$$
\begin{aligned}
& P_{G}\left(R_{1, f-1}=F, R_{2, f-1}=F, \ldots, R_{d_{f-1}^{i f}, f-1}\right. \\
& \left.\quad=F, R_{i f}=T\right)=P_{G}\left(R_{1, f-1}=F\right) P_{G}\left(R_{2, f-1}\left(14 \mathrm{~b}^{d_{f-1}^{i f}}\right)\right. \\
& \quad=F) \cdots P_{G}\left(R_{d_{f-1}^{i f}, f-1}=F\right) P_{G}\left(R_{i f}\right. \\
& \quad=T \mid R_{1, f-1}=F, R_{2, f-1}=F, \ldots, R_{d_{f-1}^{i f}, f-1} \\
& \quad=F) .
\end{aligned}
$$

Finally, according to formulas (11)- $\left(14 \mathrm{~b}^{d_{f-1}^{i f}}\right)$, the comprehensive probability $P_{i}^{G}$ of occurrence of each risk is calculated. Furthermore, according to formula (10), each value at risk $z_{i}$ is calculated.

\section{The Calculating Case for Situations A and B}

In the following part, 2 calculating cases for situations A and $\mathrm{B}$ are used to explain the RAM proposed above.

Case 1 for Situation A. In order to improve its competitive ability, Liaoning GTE Biopharmaceutical Company wants to outsource its clinical experiment business. Before outsourcing, the company needs risk assessment on this outsourcing activity. The company sets up a panel of experts including 5 experts $\left(E_{1}, E_{2}, \ldots, E_{5}\right)$, and according to experience and business proficiency of each expert, the weight vectors of experts provided by the company are $w=(0.25,0.2,0.3,0.1,0.15)$. Through relevant analysis and arrangement of feedback suggestions from questionnaires, the group of experts determine 2 kinds of outsourcing risks: bad book $\left(R_{1}\right)$ and contract modification $\left(R_{2}\right)$. These 2 risks cannot be divided to a hierarchical structure. It should be calculated by using formulas (8), (9), and (10). The 5 experts give the value as Tables 1 and 2 show.

By using formulas (8), (9), and (10), we can get

$$
\begin{aligned}
P_{1}^{G}= & \sum_{k=1}^{n} w_{k} P_{1}^{k} \\
= & 0.25 * 0.36+0.2 * 0.24+0.3 * 0.28+0.1 \\
& * 0.32+0.15 * 0.24=0.29 \\
L_{1}^{G}= & \sum_{k=1}^{n} w_{k} l_{1}^{k} \\
= & 0.25 * 20+0.2 * 22+0.3 * 25+0.1 * 22 \\
& +0.15 * 22=22.3 \\
z_{1}= & P_{1}^{G} L_{1}^{G}=0.29 * 22.3=6.467 \\
P_{2}^{G}= & \sum_{k=1}^{n} w_{k} P_{2}^{k} \\
= & 0.25 * 0.32+0.2 * 0.34+0.3 * 0.25+0.1 \\
& * 0.25+0.15 * 0.35=0.3005
\end{aligned}
$$


TABLE 1

\begin{tabular}{cccccc}
\hline & $E_{1}$ & $E_{2}$ & $E_{3}$ & $E_{4}$ & $E_{5}$ \\
\hline$P\left(R_{1}=T\right)$ & 0.36 & 0.24 & 0.28 & 0.32 & 0.24 \\
$P\left(R_{2}=T\right)$ & 0.32 & 0.34 & 0.25 & 0.25 & 0.35 \\
\hline
\end{tabular}

TABLE 2

\begin{tabular}{llllll}
\hline$l_{i}^{k}$ & $E_{1}$ & $E_{2}$ & $E_{3}$ & $E_{4}$ & $E_{5}$ \\
\hline$R_{1}$ & 20 & 22 & 25 & 22 & 22 \\
$R_{2}$ & 28 & 30 & 28 & 29 & 28 \\
\hline
\end{tabular}

$$
\begin{aligned}
L_{2}^{G}= & \sum_{k=1}^{n} w_{k} l_{2}^{k} \\
= & 0.25 * 28+0.2 * 30+0.3 * 28+0.1 * 29 \\
& \quad+0.15 * 28=28.5, \\
z_{2}= & P_{2}^{G} L_{2}^{G}=0.29 * 22.3=28.8005 .
\end{aligned}
$$

The risk value of contract modification is much bigger than the value of bad booking. The company should consider this result to design the outsourcing plan.

Case 2 for Situation B. On the same background, through relevant analysis and review of feedback suggestions from questionnaires, the panel of experts determines 5 outsourcing risks: bad book $\left(R_{1}\right)$, contract modification $\left(R_{2}\right)$, decreased service quality $\left(R_{3}\right)$, hidden costs $\left(R_{4}\right)$, and damaged company image $\left(R_{5}\right)$. The 5 risk-associated matrixes offered by the experts are as follows:

$$
\begin{gathered}
A_{1}=\begin{array}{ccccc}
R_{1} & R_{2} & R_{3} & R_{4} & R_{5} \\
R_{1} \\
R_{2} \\
R_{3} \\
R_{4} \\
R_{5}
\end{array}\left[\begin{array}{lllll}
0 & 2 & 3 & 2 & 0 \\
0 & 0 & 0 & 0 & 5 \\
0 & 0 & 0 & 0 & 0 \\
0 & 3 & 1 & 0 & 0 \\
0 & 0 & 0 & 1 & 0
\end{array}\right], \\
A_{2}=\begin{array}{l}
R_{1} \\
R_{2} \\
R_{3} \\
R_{4} \\
R_{5}
\end{array}\left[\begin{array}{llllll}
0 & 0 & 4 & 3 & 1 \\
0 & 0 & 2 & 0 & 5 \\
0 & 0 & 0 & 0 & 0 \\
0 & 3 & 1 & 0 & 0 \\
0 & 0 & 0 & 1 & 0
\end{array}\right],
\end{gathered}
$$

$$
\begin{gathered}
A_{3}=\begin{array}{l}
R_{1} \\
R_{1} \\
R_{2} \\
R_{3} \\
R_{4} \\
R_{5}
\end{array}\left[\begin{array}{lllll}
0 & 2 & 3 & 4 & 0 \\
0 & 0 & 0 & 0 & 5 \\
0 & 0 & 0 & 0 & 0 \\
0 & 3 & 1 & 0 & 0 \\
0 & 0 & 1 & 4 & 0
\end{array}\right], \\
A_{4}= \\
R_{1} \\
R_{3} \\
R_{4} \\
R_{5}
\end{gathered}\left[\begin{array}{ccccc}
0 & 0 & 5 & 3 & 0 \\
0 & 0 & 0 & 0 & 5 \\
0 & 0 & 0 & 0 & 0 \\
0 & 3 & 4 & 0 & 1 \\
0 & 0 & 0 & 1 & 0
\end{array}\right],
$$

Firstly, according to formula (1), the 5 risk-associated matrixes $\left(A_{1}, A_{2}, \ldots, A_{5}\right)$ are combined as the groupassociated matrix $A_{G}=\left[a_{i j}^{G}\right]_{5 \times 5}$ :

$$
\begin{aligned}
& \begin{array}{lllll}
R_{1} & R_{2} & R_{3} & R_{4} & R_{5}
\end{array} \\
& A_{G}=\begin{array}{c}
R_{1} \\
R_{2} \\
R_{3} \\
R_{4} \\
R_{5}
\end{array}\left[\begin{array}{ccccc}
0 & 0 & 3.7 & 2.9 & 0 \\
0 & 0 & 0 & 0 & 5 \\
0 & 0 & 0 & 0 & 0 \\
0 & 3 & 1.75 & 0 & 0 \\
0 & 0 & 0 & 0 & 0
\end{array}\right] .
\end{aligned}
$$

Secondly, according to formula (2), the group-associated matrix $A_{G}$ is transformed into regulated group-associated matrix $B_{G}=\left[b_{i j}^{G}\right]_{5 \times 5}$; namely,

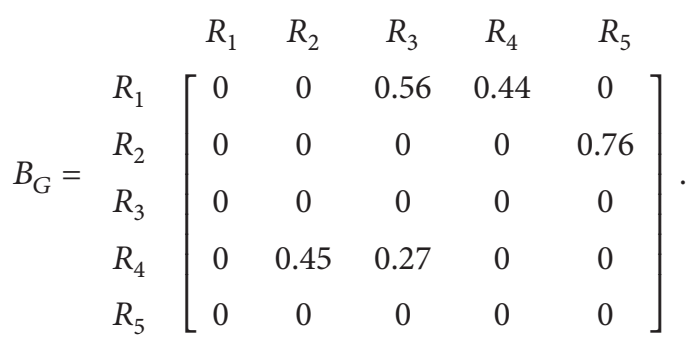




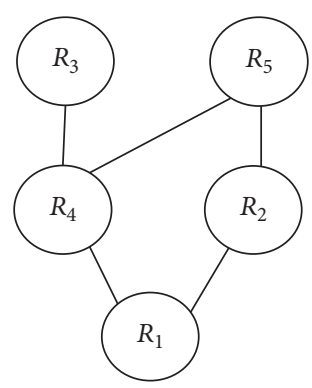

FIgURE 1: Hierarchical structure of risks.

According to formula (3), group comprehensive influence matrix $C=\left[c_{i j}\right]_{5 \times 5}$ is constructed; that is,

$$
\begin{aligned}
& \begin{array}{lllll}
R_{1} & R_{2} & R_{3} & R_{4} & R_{5}
\end{array} \\
& C=\begin{array}{c}
R_{1} \\
R_{2} \\
R_{3} \\
R_{4} \\
R_{5}
\end{array}\left[\begin{array}{ccccc}
0 & 0.198 & 0.679 & 0.44 & 0.15 \\
0 & 0 & 0 & 0 & 0.76 \\
0 & 0 & 0 & 0 & 0 \\
0 & 0.45 & 0.27 & 0 & 0.342 \\
0 & 0 & 0 & 0 & 0
\end{array}\right] .
\end{aligned}
$$

Furthermore, according to the situation of the company, the thresholds about comprehensive influence matrix provided by 5 experts separately are $\lambda_{1}=0.1, \lambda_{2}=0.1, \lambda_{3}=$ $0.15, \lambda_{4}=0.1, \lambda_{5}=0.1$. According to formula (4), the group threshold is $\lambda_{G}=0.115$. According to formula (5), the comprehensive influence matrix $C$ is transformed into $0-1$ comprehensive influence matrix $D=\left[d_{i j}\right]_{5 \times 5}$; that is,

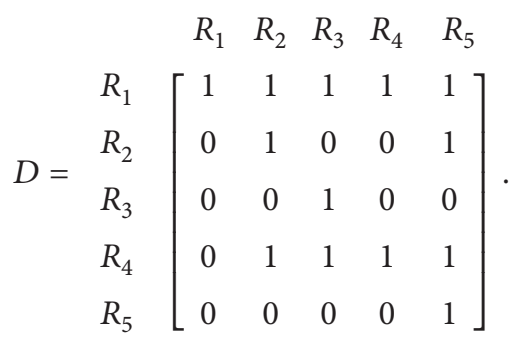

According to matrix $D$ and formula (7), the hierarchical structure of 5 kinds of risks is divided as shown in Figure 1.

According to the hierarchical structure of risks shown in Figure 1, combined with the historical data and reality of the market, the panel of experts offered the probability of occurrence of the bottom risk $R_{1}$, the conditional probability of occurrence of the risks on the second layer $R_{2}$ and $R_{4}$, and the conditional probability of occurrence of the risks on the third layer $R_{3}$ and $R_{5}$, as shown in Table 3. The loss of risks offered by experts (unit: ten thousand Yuan) is listed in Table 4. Furthermore, according to formulas (11)-(14.2 $\left.2_{f-1}^{i f}\right)$, the comprehensive probability of occurrence of risks $P_{i}^{G}$ is calculated, shown in the second list of Table 5. For example, the probability of occurrence of the risk $R_{2}$ is $P_{2}^{G}=P_{G}\left(R_{1}=\right.$ $T) P_{G}\left(R_{2}=T \mid R_{1}=T\right)+P_{G}\left(R_{1}=F\right) P_{G}\left(R_{2}=T \mid\right.$ $\left.R_{1}=F\right)=0.284$. According to formula (9), the loss of risks
TABLE 3: Probability and conditional probability of occurrence of risks offered by experts.

\begin{tabular}{lccccc}
\hline & $E_{1}$ & $E_{2}$ & $E_{3}$ & $E_{4}$ & $E_{5}$ \\
\hline$P\left(R_{1}=T\right)$ & 0.36 & 0.24 & 0.28 & 0.32 & 0.24 \\
$P\left(R_{2}=T \mid R_{1}=T\right)$ & 0.42 & 0.44 & 0.45 & 0.45 & 0.45 \\
$P\left(R_{2}=T \mid R_{1}=F\right)$ & 0.22 & 0.20 & 0.23 & 0.23 & 0.22 \\
$P\left(R_{4}=T \mid R_{1}=T\right)$ & 0.33 & 0.35 & 0.33 & 0.32 & 0.36 \\
$P\left(R_{4}=T \mid R_{1}=F\right)$ & 0.15 & 0.12 & 0.11 & 0.15 & 0.14 \\
$P\left(R_{3}=T \mid R_{4}=T\right)$ & 0.40 & 0.38 & 0.42 & 0.40 & 0.40 \\
$P\left(R_{3}=T \mid R_{4}=F\right)$ & 0.20 & 0.22 & 0.20 & 0.23 & 0.21 \\
$P\left(R_{5}=T \mid R_{2}=T, R_{4}=T\right)$ & 0.44 & 0.42 & 0.44 & 0.43 & 0.44 \\
$P\left(R_{5}=T \mid R_{2}=T, R_{4}=F\right)$ & 0.40 & 0.38 & 0.37 & 0.38 & 0.37 \\
$P\left(R_{5}=T \mid R_{2}=F, R_{4}=T\right)$ & 0.33 & 0.32 & 0.28 & 0.26 & 0.28 \\
$P\left(R_{5}=T \mid R_{2}=F, R_{4}=F\right)$ & 0.18 & 0.15 & 0.20 & 0.16 & 0.16 \\
\hline
\end{tabular}

TABLE 4: Loss of risks offered by experts.

\begin{tabular}{llllll}
\hline$l_{i}^{k}$ & $E_{1}$ & $E_{2}$ & $E_{3}$ & $E_{4}$ & $E_{5}$ \\
\hline$R_{1}$ & 20 & 22 & 25 & 22 & 22 \\
$R_{2}$ & 28 & 30 & 28 & 29 & 28 \\
$R_{3}$ & 56 & 56 & 54 & 55 & 54 \\
$R_{4}$ & 64 & 65 & 66 & 66 & 64 \\
$R_{5}$ & 80 & 78 & 78 & 80 & 82 \\
\hline
\end{tabular}

TABLE 5: Probability of occurrence of risks, loss of risks, and value at risk.

\begin{tabular}{lccc}
\hline & $P_{i}^{G}$ & $l_{i}^{G}$ & $z_{i}$ \\
\hline$R_{1}$ & 0.29 & 22.4 & 6.5 \\
$R_{2}$ & 0.284 & 28.5 & 8 \\
$R_{3}$ & 0.245 & 55 & 13.5 \\
$R_{4}$ & 0.19 & 65 & 12.4 \\
$R_{5}$ & 0.253 & 79.3 & 20 \\
\hline
\end{tabular}

$\left(l_{i}^{1}, l_{i}^{2}, \ldots, l_{i}^{5}\right)$ is combined as the comprehensive loss of risks $L_{i}^{G}$, as shown in the third list of Table 3. Finally, according to formula (10), each value at risk $z_{i}$ is calculated as shown in fourth list of Table 3. Thus, the calculating results of risk assessment provide decision support for risk management of the biopharmaceutical company.

\section{Conclusion}

A risk assessment method in consideration of risk-associated situations is provided in this paper. Based on various kinds of evaluating information about risks offered by the group of experts, this method calculates each value at risk through identification of hierarchical structure of risks by adopting knowledge related to probability theory. According to calculation analysis, the proposed method is feasible and proved to have certain application value. For structured risk assessment problems, the proposed method is general. However, the requirements of different industries and different types of business for service outsourcing are not the same. So the method we give in this paper may not be directly applicable 
to all industries or all types of enterprises, especially in some special industries. As service outsourcing progresses, some of the expected outsourcing risks will change, and on the other hand, new and unpredictable outsourcing risks will emerge. In order to ensure the smooth realization of service outsourcing, the enterprise risk control during the whole service outsourcing process is worth studying. Also further research is required for large calculation amount of the risk occurrence.

\section{Competing Interests}

The authors declare that there is no conflict of interests regarding the publication of this paper.

\section{References}

[1] M. Doumpos and C. Zopounidis, "Assessing financial risks using a multicriteria sorting procedure: the case of country risk assessment," Omega, vol. 29, no. 1, pp. 97-109, 2001.

[2] T. M. Williams, "Risk-management infrastructures," International Journal of Project Management, vol. 11, no. 1, pp. 5-10, 1993.

[3] S. Piramuthu, "On preprocessing data for financial credit risk evaluation," Expert Systems with Applications, vol. 30, no. 3, pp. 489-497, 2006.

[4] M. Farrell and R. Gallagher, "The valuation implications of enterprise risk management maturity," Journal of Risk and Insurance, vol. 82, no. 3, pp. 625-657, 2015.

[5] J. C. Wei, Z. J. Li, L. Q. Shi et al., "Comprehensive evaluation of water-inrush risk from coal floors," Mining Science and Technology (China), vol. 20, no. 1, pp. 121-125, 2010.

[6] M. Nazam, J. Xu, Z. Tao, J. Ahmad, and M. Hashim, "A fuzzy AHP-TOPSIS framework for the risk assessment of green supply chain implementation in the textile industry," International Journal of Supply and Operations Management, vol. 2, no. 1, pp. 548-568, 2015.

[7] B. Bahli and S. Rivard, "Validating measures of information technology outsourcing risk factors," Omega, vol. 33, no. 2, pp. 175-187, 2005.

[8] C. Oksel, C. Y. Ma, and X. Z. Wang, "Structure-activity relationship models for hazard assessment and risk management of engineered nanomaterials," Procedia Engineering, vol. 102, no. 1, pp. 1500-1510, 2015.

[9] K. L. Astles, M. G. Holloway, A. Steffe, M. Green, C. Ganassin, and P. J. Gibbs, "An ecological method for qualitative risk assessment and its use in the management of fisheries in New South Wales, Australia," Fisheries Research, vol. 82, no. 1-3, pp. 290-303, 2006.

[10] A. S. Markowski and M. Sam Mannan, "Fuzzy risk matrix," Journal of Hazardous Materials, vol. 159, no. 1, pp. 152-157, 2008.

[11] D. Donoho and M. Gavish, "Minimax risk of matrix denoising by singular value thresholding," The Annals of Statistics, vol. 42, no. 6, pp. 2413-2440, 2014.

[12] A. Khashman, "Neural networks for credit risk evaluation: investigation of different neural models and learning schemes," Expert Systems with Applications, vol. 37, no. 9, pp. 6233-6239, 2010.

[13] E. Angelini, G. Tollo, and A. Roli, "A neural network approach for credit risk evaluation," The Quarterly Review of Economics and Finance, vol. 48, no. 4, pp. 733-755, 2008.
[14] E. G. Baranoff and T. W. Sager, "The relations among asset risk, product risk, and capital in the life insurance industry," Journal of Banking \& Finance, vol. 26, no. 6, pp. 1181-1197, 2002.

[15] M. Abdellaoui, P. Klibanoff, and L. Placido, "Experiments on compound risk in relation to simple risk and to ambiguity," Management Science, vol. 61, no. 6, pp. 1306-1322, 2015.

[16] X.-W. Liao, W. Tao, and L. Yuan, "A Bayesian network model under group decision making for evaluating IT outsourcing risk," in Proceedings of the International Conference on Risk Management \& Engineering Management (ICRMEM '08), pp. 559-564, Beijing, China, November 2008.

[17] G. Büyüközkan and D. Ruan, "Choquet integral based aggregation approach to software development risk assessment," Information Sciences, vol. 180, no. 3, pp. 441-451, 2010. 


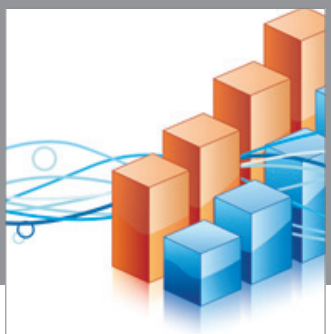

Advances in

Operations Research

vatem alat4

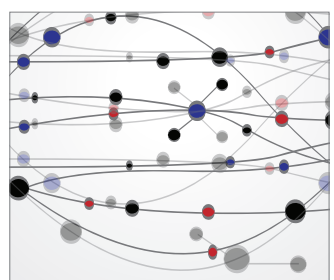

\section{The Scientific} World Journal
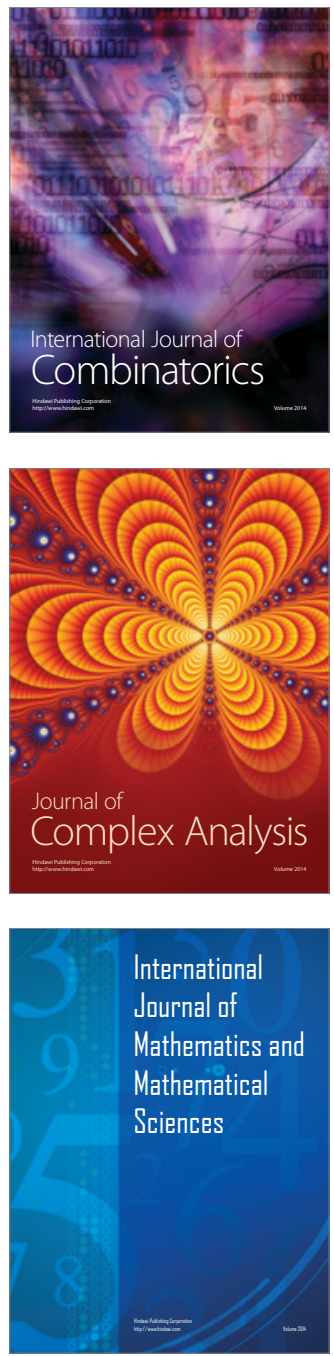
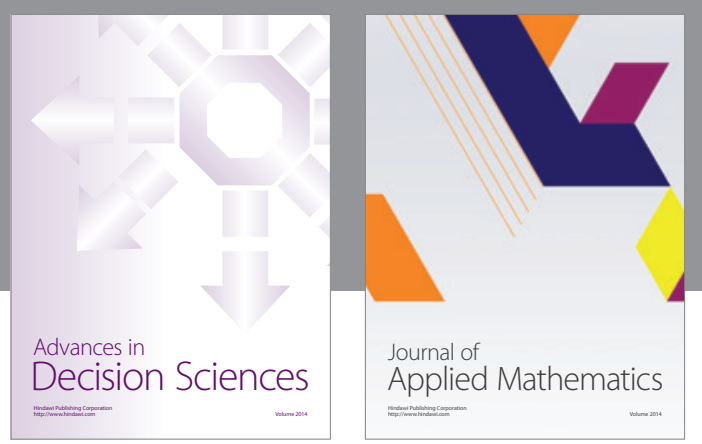

Algebra

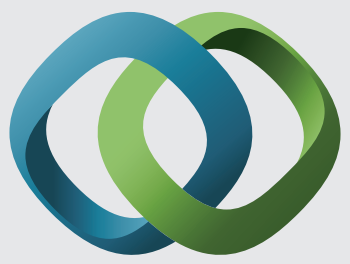

\section{Hindawi}

Submit your manuscripts at

http://www.hindawi.com
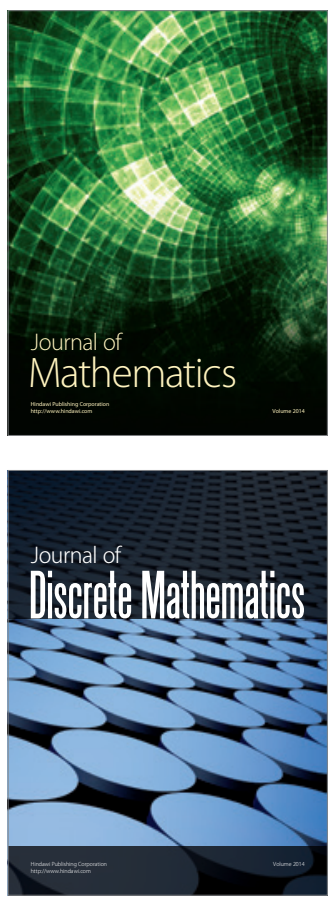

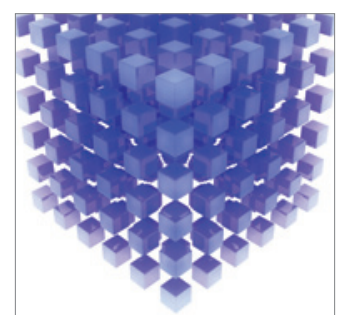

Mathematical Problems in Engineering
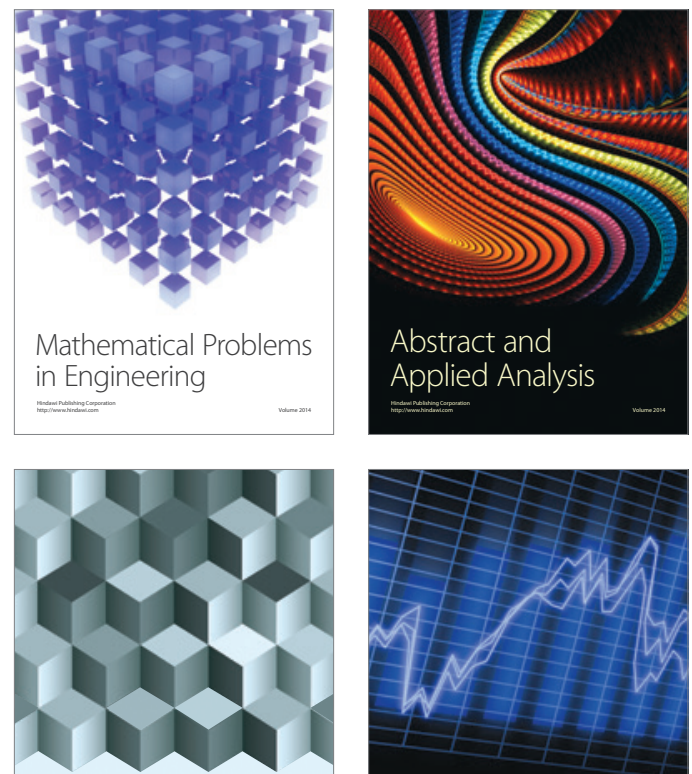

Journal of

Function Spaces

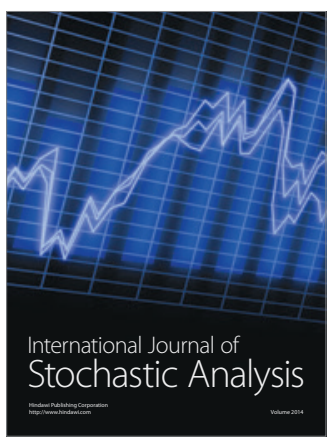

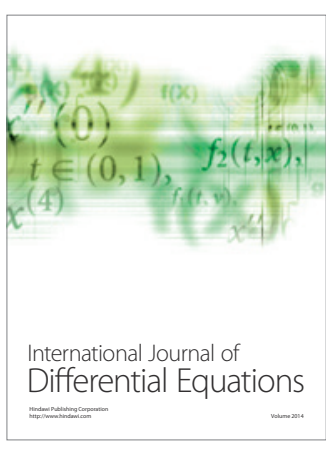
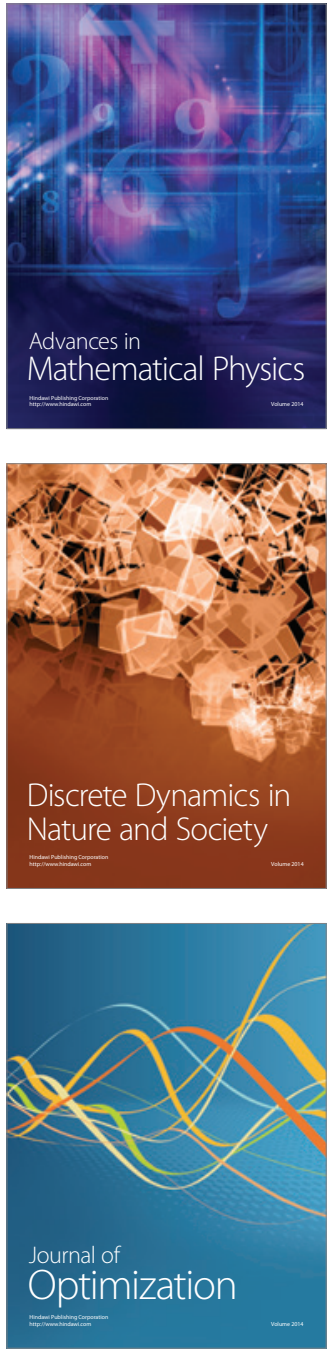